\title{
The Open Systems Industry and the Lack of Open Distributed Management
}

\author{
Lance Travis \\ Open Software Foundation,11 Cambridge Center, Cambridge, MA 02142, USA \\ cmt@osf.org

\section{Abstract}

This paper discusses reasons why the Open Systems industry has failed in its attempts to deliver interoperable vendor-independent distributed systems and network management products.

\author{
Keyword Codes: K.6.3; K.6.4 \\ Keywords: Software Management, System Management
}

\section{INTRODUCTION}

The Open Systems industry has not provided a strong response to user needs for open distributed management. End users are quite clear in their requirements. They are asking for management systems that allow them to manage their entire enterprise. They have systems from multiple vendors running many different operating systems, networking protocols, and applications. They want to be able to manage the different components with one set of tools. In addition, they want to be able to integrate and manage new technologies without abandoning their legacy systems.

Currently, they have to buy management applications tailored to each individual platform. These applications cannot share data with other similar applications for other platforms and the end result is that end-users have to buy, learn, and use multiple dissimilar applications in order to manage their enterprise. Additionally, they are not able to manage parts of their enterprise because management applications don't exist.

The end-users believe that the Open Systems industry should provide the focal point for solving these problems. By having systems vendors and software vendors agree on standards for programming interfaces and management protocols, end-users hope to be able to buy management products that can be used to manage their entire enterprise and that can be used with other vendor's management products to solve more complex management problems. For example, in the ideal world of open systems management, an enduser should be able to buy a software distribution application that can be used to install applications from any other vendor and can be used to install applications on any target system. The software distribution application should be able to share data with configuration and licensing applications so that the user can have a basis for sophisticated asset management. Unfortunately, current products do not support this ideal. Instead, end-users can buy software distribution applications from a multitude of vendors that can be used on a limited set of platforms (usually only those of the vendor) and can only be used to install a limited set of applications (usually those of the vendors).

The remainder of this paper examines why standards efforts and the multiple vendor consortium investigating open management have failed to deliver much substance. The final section provides criteria necessary for a successful delivery of open systems distributed management.

\section{STANDARDS}

Lack of standards has not been the only cause of this problem. In some management areas, more than enough standards exist. In the software distribution example, PASC (POSIX) standards exist for packaging software and for the interfaces for a software distribution applications and standards exist for network connectivity, naming, and security. 
This suite of standards should enable the ideal open systems software distribution application to exist, but the application does not exist. Obviously more than just standards need to exist.

Standards do not completely solve the problem for multiple reasons. Too many uncoordinated groups are working on standards in the management area. The Desktop Management Taskforce (DMTF), X/Open, Network Management Forum (NMF), Management

Interface Consortium (MIC), OSF, OMG, ISO, and IETF are all working on various sets of management related standards. Little formal or informal coordination exists between these organizations, which results in multiple models for defining management data (MIBs, MIFs, GDMO, OMG IDL) and mismatched management models. Vendors are forced to track and participate in multiple forums and end-users are confused as to which standards are important. Some vendors seem to be more interested in issuing press releases that extol their commitment to open systems by pointing to the large number of standards activities in which they participate than they are in actually implementing technology based on the standards.

SNMP has succeeded as the starting point for many management applications and the industry has been able to quickly develop a set of interoperable SNMP-based technologies. The problems associated with poorly coordinated efforts have begun to infiltrate the SNMP community. Many groups have begun to define private SNMP-based MIBS for their devices or applications. Unfortunately, these extensions to the official standard MIBs create problems for the application vendors since the applications must be aware of the extensions in order to make use of them. The groups defining different MIBs align their efforts with a small number of application vendors (or are themselves the application vendors). Unfortunately, other applications that can process SNMP data are not aware of the extensions and users now must by multiple SNMP-based management applications in order to manage the MIB extensions.

In other areas, standards exist, but do not cover the entire problem space. For example, for network management SNMP and CMIP and their related management object models define how to define and move management data. XMP provides a standard API for accessing this data. Many management platforms support these standards, but unfortunately, each vendor adds their own user interface development tools (e.g., OpenView Windows), event processing, name space management, and their means managing the management data databases and eliminate the portability and interoperability that that standards begin to provide. Also, standards exist for managing software license databases, but no standards exist for an API allowing applications to access a license database or for the format of the database.

Standards by themselves are not sufficient to guarantee interoperability. No matter how precise the definition of a protocol, two different implementors will create implementations that will not interoperate unless they cooperate during the implementation. Different interpretations of written text and inevitable holes in the specification will result in non-interoperable implementations. At a minimum, conformance and interoperability tests are required to ensure that implementations derived from a standard will interoperate. Unfortunately, the cost of developing such tests is high and the organization that creates the standard usually does not have the resources or charter to do such tests and they never get implemented. Some organizations such as COS, rely on donated tests for their interoperability certification. Since vendors have little incentive to incur the cost of developing this tests alone, few tests get donated. 


\section{VENDOR CONSORTIUM}

Some vendors have recognized the problem of relying solely on standards to create open interoperable solutions and have formed consortium and joint development efforts in order to develop shared technologies as the basis of interoperable solutions. A common starting point for vendor products can greatly accelerate the development of interoperable products. For example, the release on TCP/IP source code as part of the BSD 4.2 release of Unix greatly expanded the use and deployment of TCP/IP. Vendors had a way to quickly implement the technology and were able to check interoperability against other implementations based on the same starting point. The original authors of the SNMP specification each implemented a version of the technology, resolved interoperability problems among their separate implementations and then licensed this technology to the world. Shortly after this, two free versions of SNMP became available on the Internet. Within two years, SNMP became the dominant network management protocol and interoperability problems among different vendor's products were virtually nonexistent. (The proliferation of non-standard and private MIBs has created problems within the SNMP community.) CMIP or CMOT for which no reference implementation was ever produced is virtually non-existent in LAN management.

Within the management area, several consortium have failed to deliver technology that can be used as the basis for a set of management products. Unix International ceased to exist long before the UI Atlas management platform became available. The companies working under the COSE process on system management never delivered any of the system management technology they announced two years ago and they have stopped trying. The OSF DME program was greatly scaled back and its system management and network management technology is being used by only a few of the OSF sponsor companies that funded its development. The DMTF was beginning to make progress but Microsoft has reduced their commitment to the consortium. For a consortium effort to be successful, vendors need to learn how to cooperate with their traditional rivals. Self interest can easily derail these efforts.

The cause of these failures seems to be the result of each vendor's concerns over protecting their installed base and maintaining account control. Vendors have invested greatly in developing their own management products. Replacing these products with products based on a common reference technology becomes very difficult unless tools and transition aids are provided that will allow the vendors existing installed based to interoperate with the new technologies. Since each vendors installed based widely diverges from the other vendors, efforts to agree on common technologies quickly become bogged down with endless discussions about whose programming interfaces and management model to preserve.

\section{NECESSARY CONDITIONS}

More than these numerous ad hoc standards and consortium efforts are needed. All of the following four items are needed to help drive the open systems industry towards complete interoperable management solutions. 
- A strong coordinated effort for developing a complete suite of specifications. Individual management specification efforts (e.g., X/Open, OMG, MIC and others) should continue to develop the management models and specifications for their particular niches and areas of interest, but they should work to ensure that their work fits into a large enterprise mode for management. The Network Management Forum's (NMF) OMNIPoint specification is a good starting point for a complete reference model for management.

- Reference implementations based on the emerging specifications that can be used by vendors as the starting point for their products. This reference implementation needs to be easily accessible and should not reflect any specific vendor bias. The OSF prestructured technology (PST) process is designed to allow vendors to work cooperatively on reference software. Teaching vendors how to work together cooperatively on joint projects is a very difficult task and is a focal point of the PSTG process. The reference implementations will have to provide the flexibility in order to allow for the integration of existing management applications.

- Formal specifications, conformance testing, and certification programs based on the reference implementations. X/Open has started this work for some of their technologies. A valid economic model for the creation of tests needs to be found.

- End user purchasing profiles to force vendors to make the products. End-users need to back their purchasing profiles and purchase specifications with their money. Vendors are continuing to make money selling their existing management products and promoting their proprietary management interfaces and their non-interoperable solutions. The vendors have little financial incentive to change unless the end-users force the issue by not buying the existing products. This may be the most important factor of all. If users specify open distributed management products but continue to purchase vendor proprietary products, the vendors will never build the open management products.

If any of the above four are missing (which is today's situation) the open systems industry will continue to fail to deliver open distributed management solutions. 